\title{
Progress towards elimination of trans-fatty acids in foods commonly consumed in four Latin American cities
}

\author{
Rafael Monge-Rojas ${ }^{1, *}$, Uriyoán Colón-Ramos ${ }^{2}$, Enrique Jacoby ${ }^{3} \uparrow$, Thelma Alfaro ${ }^{1}$, \\ Maria das Graças Tavares do Carmo ${ }^{4}$, Salvador Villalpando ${ }^{5}$ and Claudio Bernal ${ }^{6}$ \\ ${ }^{1}$ Costa Rican Institute for Research and Education on Nutrition and Health (INCIENSA), Ministry of Health, Tres Ríos, \\ Costa Rica: ${ }^{2}$ Department of Global Health, Milken Institute School of Public Health, George Washington University, \\ Washington, DC, USA: ${ }^{3}$ Pan American Health Organization/World Health Organization, Washington, DC, USA: \\ ${ }^{4}$ Instituto de Nutrição Josué de Castro, Universidade Federal do Rio de Janeiro, Rio de Janeiro, Brazil: ${ }^{5}$ Centro de \\ Investigación en Nutrición y Salud, Instituto Nacional de Salud Pública, Cuernavaca, Mor, México: ${ }^{6}$ Cátedra \\ Bromatología y Nutrición, Facultad de Bioquímica y Ciencias Biológicas, Universidad Nacional del Litoral, \\ Santa Fe, Argentina
}

Submitted 3 February 2017: Final revision received 10 April 2017: Accepted 15 May 2017: First published online 23 June 2017

\begin{abstract}
Objective: To assess progress towards the elimination of trans-fatty acids (TFA) in foods after the 2008 Pan American Health Organization (PAHO) recommendation of virtual elimination of TFA in Latin America.

Design: A descriptive, comparative analysis of foods that were likely to contain TFA and were commonly consumed in four cities in Latin America.

Setting: San José (Costa Rica), Mexico City (Mexico), Rio de Janeiro (Brazil), Buenos Aires (Argentina).

Subjects: Foods from each city were sampled in 2011; TFA content was analysed using GC. TFA of selected foods was also monitored in 2016.

Results: In 2011-2016, there was a significant decrease in the content of TFA in the sampled foods across all sites, particularly in Buenos Aires (from 12.6-34.8\% range in 2011-2012 to nearly 0\% in 2015-2016). All sample products met the recommended levels of TFA content set by the PAHO. TFA were replaced with a mixture of saturated and unsaturated fats.

Conclusions: Our results indicate a virtual elimination of TFA from major food sources in the cities studied. This could be due to a combination of factors, including recommendations by national and global public health authorities, voluntary and/or mandatory food reformulation made by the food industry.
\end{abstract}

Keywords
rans-fatty acids
Food samples
Latin America
The positive health impact of reducing or even eliminating industrially produced trans-fatty acids (IP-TFA) is undisputed. There is conclusive evidence that consumption of IP-TFA increases CHD risk factors and CHD events, as well as risk of fatal CHD and sudden cardiac death, diabetes, and components of the metabolic syndrome ${ }^{(1,2)}$.

Because IP-TFA pose a serious threat to human health, their elimination from the food supply is a public health priority in various countries ${ }^{(3)}$. In line with these priorities, in 2003 the FAO and WHO recommended that intake of both industrially and ruminant-produced trans-fatty acids (TFA) should be less than $1 \%$ of the daily dietary energy intake ${ }^{(4)}$. In 2010, the FAO/WHO Expert Consultation report advocated for more leniency in the recommendation to protect

† Present affiliation: Núcleo de Pesquisas Epidemiológicas em Nutrição e Saúde (NUPENS), Universidade de São Paulo, São Paulo, Brazil. vulnerable consumers who were at risk of consuming too much TFA (i.e. young adults, teenagers, children and lowincome individuals). This led to the recommendation to remove partially hydrogenated fats and oils (also known as IP-TFA) from the food supply ${ }^{(4)}$.

National and local bans of IP-TFA are aimed to nudge industry to reformulate its products in order to virtually eliminate IP-TFA in its foodstuffs ${ }^{(5,6)}$, but progress towards this elimination has been sparsely monitored ${ }^{(5,7-10)}$. In Latin America, various governments have taken action since 2005 to reduce IP-TFA content in foods ${ }^{(11)}$. Some of these actions have been reinforced by the Pan American Health Organization (PAHO)/WHO Trans Fat Free Americas Task Force, which encouraged countries to pass legislation to limit IP-TFA intake ${ }^{(12)}$. Major food companies in the Latin American region also supported this mission to eliminate IP-TFA voluntarily from their foodstuffs ${ }^{(13)}$. 
However, evidence up to 2009 showed limited and inconsistent progress towards this elimination ${ }^{(3)}$.

The current study seeks to update this progress by presenting the results of monitoring efforts of IP-TFA content in foods in large cities of four Latin American countries. Data are from the period 2011-2016 and report if these countries abided by the PAHO/WHO recommendation to limit TFA to $<2 \%$ of the total fat amount in vegetable oil and spreadable margarines, and to $<5 \%$ for any other processed foods, to reduce IP-TFA ${ }^{(12)}$. Since 2005, the four Latin American countries used various strategies to reduce IP-TFA, including a ban or limit on IP-TFA content, mandatory labelling, voluntary regulation by the food industry and consumer education efforts ${ }^{(11)}$. Specifically, Brazil had a legislation since 2007 that prohibited the process of hydrogenation of vegetable oil and made food companies declare the TFA content of their pre-packaged foodstuffs ${ }^{(11)}$. In Mexico and Costa Rica, there has been no legislation to limit TFA content, but since 2010 companies must declare TFA content if they desire to make a claim with respect to fat or cholesterol content of a product ${ }^{(14,15)}$. Finally, towards the end of year 2014, Argentina started to enforce its legislation to limit IP-TFA content to no more than $2 \%$ of total fats in vegetable oils and margarines and to no more than $5 \%$ of total fats in other foods ${ }^{(16)}$.

The information obtained from continuous monitoring of IP-TFA in these and other countries is a critical step in the discussion of effective strategies to virtually eliminate these fatty acids from the food supply. Lessons gleaned from this monitoring can be applied to future intersectoral collaborations (e.g. government, academy, food industry, consumers) that subscribe to the mission of improving the nutritional profiles of pre-packaged foods with the ultimate goal of improving the health of consumers.

\section{Methods}

\section{Study design}

Data are from a multicentre study designed to collect data from foodstuffs that were known to contain TFA and were also commonly consumed in four major cities in Latin America. Data were collected during two time periods (2011-2012 and 2015-2016). The cities of San José in Costa Rica, Mexico City in Mexico, Rio de Janeiro in Brazil and Buenos Aires in Argentina were chosen for the present study by two criteria: (i) they represented four major cities of countries in North, Central and South America; and (ii) they had the resources, laboratory space and personnel to collect and analyse the data. All data collection instruments and analytical techniques were standardized across the data collection sites in each city following the directions detailed in the multicentre study protocol $^{(17)}$. All participating laboratories were able to apply the same analytical techniques and reproduce results with acceptable reliability and reproducibility ( $Z$-scores $\leq 2$ ). $Z$-scores were determined according to the ISO/IEC 43-1 guideline ${ }^{(18)}$. More details about the methodology can be found in the official protocol of the multicentre study ${ }^{(17)}$.

In brief, the study protocol followed three consecutive stages for data collection.

First, an open-ended food survey was designed to ask about the types, brands and place of purchase of the following foods: spreadable fats (margarines), edible oils, pastries; cookies (simple/plain cookies, cookies with artificially flavoured cream (filled), cookies covered with chocolate); fast foods (French fries) of the three major transnational fast-food chains (McDonald's, Burger King, Kentucky Fried Chicken (KFC)); and fast foods prepared in either restaurants or food shacks (fried corn flakes, potato chips, corn-based foods and popcorn, among others).

Second, two counties within each city were selected according to their socio-economic profile (average household income, educational attainment and occupations). This profile had to correspond to the most prevalent socio-economic level in the entire city. Within this criterion, the first county was selected at random and the second county was selected at random among the counties that bordered the initial county. About 400 individuals were randomly selected in each city to complete the survey.

Third, food samples of the types and brands of foods commonly consumed in each city were collected (as per the results of the food consumption survey). We obtained samples of the three types and brands of foodstuffs most commonly consumed by the sampled population in each city. For example, for margarines, we obtained samples of three types of margarines and three brands most commonly consumed in each city. Each sample consisted of five units of margarine, for example, each coming from the same production batch. One unit was equivalent to one package, bottle, stick or any of the regular formats of the food item. For non-pre-packaged foods, such as fast foods and pastries, the investigators bought five units of the prepared foods in three different restaurants or pastry shops of the same chain. These units were then conglomerated to make one composite sample. Sampling was performed following the guidelines established by the International Organization of Legal Metrology ${ }^{(19)}$.

To obtain a composite sample, the five units of each food were homogenized following the methodology described by the Association of Official Analytical Chemists (AOAC) ${ }^{(20)}$. An aliquot of the mixture was stored in a $10 \mathrm{ml}$ glass vial with a Teflon cap and frozen at $-20^{\circ} \mathrm{C}$ until the fatty acid compositional analysis.

Fatty acid composition of the foods was determined by GC following the methods recommended by the AOAC for the specific type of food. Extractions, methylation, identification and quantification of fatty acids were also completed following the official method AOAC $996.06^{(20)}$. All sample analyses were replicated three times. 
At all times the methodology for collection, processing and analysis was governed by the provision of the official protocol of the multicentre study.

A total of eighty-one food composite samples were analysed in every city in the 2011-2012 period. We continued to monitor the IP-TFA content of these foods during the years 2013-2014. The observed results showed the same trend in IP-TFA content; for this reason, for the period 2015-2016, a similar methodology was followed except that only those foods that were found to be high in TFA in 2013-2014 were sampled again.

\section{Data analysis}

One-factor ANOVA was used to determine the differences in the mean contents of fatty acids in the same food groups between the four cities included in the study ( $F$ statistic $<0 \cdot 05$ ). Tukey's test was used post boc for multiple comparisons.

A paired $t$ test was used to compare the differences in the fatty acid content and TFA as a percentage of total fatty acids between the periods 2011-2012 and 2015-2016.

\section{Results}

A total of 324 composite food samples were analysed for the 2011-2012 period and twenty-seven composite samples in the 2015-2016 period. We present IP-TFA content in Tables 1-6.

The total IP-TFA as a percentage of total fatty acids in 2011-2012 is shown in Table 2. The IP-TFA content varied across each city and foodstuff, with Buenos Aires reporting the highest proportion of IP-TFA in regular margarine, chocolate-filled or covered cookies and local fast foods compared with similar foodstuffs available elsewhere. But the content of IP-TFA in other food items was higher in other countries: for example, in Rio de Janeiro, it was the artificially flavoured cream-filled cookies, and in Mexico City, it was the corn snacks and the French fries from transnational chains that had significantly higher mean IP-TFA.

The average content of IP-TFA in all edible oils in 2011-2012 complied with the PAHO/WHO recommendation $(<2 \%$ of the total fat amount in vegetable oil and spreadable margarines). However, in Buenos Aires and Rio de Janeiro one of the margarine brands analysed exceeded by far the PAHO/WHO recommendation of $<2 \%$ of total fat, with IP-TFA content at 34.8 and $9.46 \%$ of total fat, respectively.

In some brands of pre-packaged cookies available in Rio de Janeiro, particularly those cookies filled with chocolate, the percentage of IP-TFA was near the upper limit of the PAHO/WHO recommendation of $<5 \%$ of total fat. The chocolate-filled or covered cookies also had the most content of IP-TFA in San José, where one brand of this type contained $8.04 \%$, and in Buenos Aires another brand had $12.0 \%$. The proportion of IP-TFA in pastries ranged between 0 and $21.2 \%$, and was especially high in pastries from Mexico City (cinnamon rolls) and Buenos Aires (butter crescents).

Generally, the percentage of IP-TFA in local fast foods was $<5 \%$ of the total fat in the product. The content of IP-TFA in French fries of transnational chains of restaurants varied considerably among countries. Out of the total fat in French fries of all transnational chains of restaurants studied, between 60 and $70 \%$ were unsaturated fatty acids, particularly oleic and linoleic acids.

The IP-TFA contents during the periods 2013-2014 and 2011-2012 were very similar.

Table 1 Mean content (grams) of trans-fatty acids per $100 \mathrm{~g}$ of foods frequently consumed in four Latin American cities, 2011-2012

\begin{tabular}{|c|c|c|c|c|c|c|c|c|c|c|}
\hline \multirow[b]{2}{*}{ Food category } & \multicolumn{2}{|c|}{$\begin{array}{l}\text { Buenos Aires, } \\
\text { Argentina }\end{array}$} & \multicolumn{2}{|c|}{$\begin{array}{c}\text { Rio de Janeiro, } \\
\text { Brazil }\end{array}$} & \multicolumn{2}{|c|}{$\begin{array}{l}\text { San José, } \\
\text { Costa Rica }\end{array}$} & \multicolumn{2}{|c|}{$\begin{array}{l}\text { Mexico City, } \\
\text { Mexico }\end{array}$} & \multirow[b]{2}{*}{$F$ value } & \multirow{2}{*}{$\begin{array}{c}\text { Statistical } \\
\text { significance } \\
\text { level }^{*}\end{array}$} \\
\hline & Mean & SD & Mean & SD & Mean & SD & Mean & SD & & \\
\hline Edible oils & 0.167 & 0.042 & 0.200 & 0.07 & 0.547 & 0.15 & 0.340 & 0.07 & $198 \cdot 849$ & 0.003 \\
\hline Soyabean oil & \multicolumn{2}{|c|}{ INA† } & 0.190 & 0.09 & 0.415 & 0.03 & 0.345 & 0.11 & 3.567 & 0.16 \\
\hline Regular margarine & $14 \cdot 810^{*}$ & $9 \cdot 63$ & $2 \cdot 257$ & 3.02 & 0.327 & 0.09 & 0.727 & 0.57 & 361.646 & 0.000 \\
\hline Pre-packaged cookies (plain) & $0.333^{*}$ & 0.06 & 0.070 & 0.03 & 0.107 & 0.06 & 0.023 & 0.06 & $322 \cdot 881$ & 0.000 \\
\hline $\begin{array}{l}\text { Pre-packaged artificially flavoured } \\
\text { cream-filled cookies }\end{array}$ & 0.231 & 0.02 & $1 \cdot 820^{*}$ & 0.87 & 0.333 & 0.37 & 0.950 & $1 \cdot 17$ & $425 \cdot 992$ & 0.000 \\
\hline $\begin{array}{l}\text { Pre-packaged chocolate-filled or } \\
\text { covered cookies }\end{array}$ & $1 \cdot 134^{*}$ & 0.09 & \multicolumn{2}{|c|}{ INA† } & 0.935 & $0 \cdot 34$ & 0.350 & $0 \cdot 11$ & 3.587 & 0.000 \\
\hline Pre-packaged corn chip snacks & 0.320 & 0.22 & 0.060 & 0.01 & 0.142 & 0.03 & $1 \cdot 317^{*}$ & 0.07 & $421 \cdot 794$ & 0.001 \\
\hline Pre-packaged potato chip snacks & 0.685 & 0.02 & $0.145^{\star}$ & 0.03 & 0.705 & 0.04 & 0.139 & 0.02 & $145 \cdot 280$ & 0.001 \\
\hline Pre-packaged pastries & $2 \cdot 200^{*}$ & 1.07 & 0.012 & 0.001 & 0.410 & 0.03 & 0.330 & 0.02 & 334.457 & 0.000 \\
\hline \multicolumn{11}{|l|}{ French fries from international chains } \\
\hline McDonald's & $0 \cdot 110$ & 0.14 & 0.070 & 0.03 & 0.295 & 0.02 & $2 \cdot 310^{\star}$ & 0.12 & 528.962 & 0.000 \\
\hline KFC & \multicolumn{2}{|c|}{ INA† } & \multicolumn{2}{|c|}{ INA† } & 0.360 & 0.42 & $4 \cdot 305^{\star}$ & 0.27 & $399 \cdot 821$ & 0.002 \\
\hline Burger King & $0 \cdot 200$ & 0.13 & \multicolumn{2}{|c|}{ INA† } & 0.055 & 0.02 & $1 \cdot 710^{\star}$ & 0.12 & $137 \cdot 521$ & 0.001 \\
\hline
\end{tabular}

*Value significantly higher compared with that of similar foods available in other Latin American countries determined by ANOVA.

†INA, information not available because according to the survey carried out, this food is not usually consumed in that country. 
Table 2 Total industrially produced trans-fatty acids (IP-TFA) as a percentage of total fatty acids in foods frequently consumed in four Latin American cities, 2011-2012

\begin{tabular}{|c|c|c|c|c|c|c|c|c|}
\hline Category & $\begin{array}{l}\text { Buenos Aires, } \\
\text { Argentina }\end{array}$ & $\%$ & $\begin{array}{l}\text { Rio de Janeiro, } \\
\text { Brazil }\end{array}$ & $\%$ & $\begin{array}{l}\text { San José, } \\
\text { Costa Rica }\end{array}$ & $\%$ & $\begin{array}{l}\text { Mexico City, } \\
\text { Mexico }\end{array}$ & $\%$ \\
\hline Edible oils & $\begin{array}{l}\text { Soyabean oil }+25 \% \text { olive oil } \\
\text { Corn oil } \\
\text { Sunflower oil }\end{array}$ & $\begin{array}{l}0.00 \\
0.14 \\
0.13\end{array}$ & $\begin{array}{l}\text { Soyabean oil, brand } 1 \\
\text { Soyabean oil, brand } 2 \\
\text { Olive oil }\end{array}$ & $\begin{array}{l}0.12 \\
0.29 \\
0.00\end{array}$ & $\begin{array}{l}\text { Soyabean oil } \\
\text { Sunflower oil } \\
\text { Soyabean oil + palm oil }\end{array}$ & $\begin{array}{l}0.40 \\
0.71 \\
0.66\end{array}$ & $\begin{array}{l}\text { Soyabean oil, brand } 1 \\
\text { Canola + sunflower oil } \\
\text { Soyabean oil, brand } 2\end{array}$ & $\begin{array}{l}0.42 \\
0.33 \\
0.27\end{array}$ \\
\hline Margarines & $\begin{array}{l}\text { Regular, brand } 1 \\
\text { Regular, brand } 2^{*} \\
\text { Regular, brand } 3\end{array}$ & $\begin{array}{r}0.12 \\
34.80 \\
1.52\end{array}$ & $\begin{array}{l}\text { Regular, brand } 1 \\
\text { Regular, brand } 2 \\
\text { Regular, brand } 3^{\star}\end{array}$ & $\begin{array}{l}0.90 \\
0.56 \\
9.46\end{array}$ & $\begin{array}{l}\text { Regular, brand } 1 \\
\text { Soft, brand } 1 \\
\text { Light, brand } 1^{\star}\end{array}$ & $\begin{array}{l}0.46 \\
1.89 \\
2.25\end{array}$ & $\begin{array}{l}\text { Regular, brand } 1 \\
\text { Regular, brand } 2 \\
\text { Regular, brand } 3\end{array}$ & $\begin{array}{l}0.00 \\
0 \cdot 13 \\
1 \cdot 38\end{array}$ \\
\hline $\begin{array}{l}\text { Pre-packaged } \\
\text { cookies (plain) }\end{array}$ & $\begin{array}{l}\text { Brand } 1 \\
\text { Brand } 2 \\
\text { Brand } 3\end{array}$ & $\begin{array}{l}0.00 \\
0.00 \\
0.00\end{array}$ & $\begin{array}{l}\text { Brand } 1 \\
\text { Brand } 2 \\
\text { Brand } 3 \\
\text { Brand } 4\end{array}$ & $\begin{array}{l}0.00 \\
0.65 \\
0.06 \\
0.21\end{array}$ & $\begin{array}{l}\text { Brand } 1 \\
\text { Brand } 2 \\
\text { Brand } 3\end{array}$ & $\begin{array}{l}0.63 \\
0.65 \\
0.47\end{array}$ & $\begin{array}{l}\text { Brand } 1 \\
\text { Brand } 2 \\
\text { Brand } 3\end{array}$ & $\begin{array}{l}0.51 \\
0.23 \\
0.25\end{array}$ \\
\hline $\begin{array}{l}\text { Pre-packaged } \\
\text { artificially flavoured } \\
\text { cream-filled cookies }\end{array}$ & $\begin{array}{l}\text { Brand } 1 \\
\text { Brand } 2 \\
\text { Brand } 3\end{array}$ & $\begin{array}{l}0.81 \\
0.77 \\
1.55\end{array}$ & $\begin{array}{l}\text { Brand } 1 \\
\text { Brand } 2 \\
\text { Brand 3† }\end{array}$ & $\begin{array}{r}4.73 \\
1.00 \\
23 \cdot 30\end{array}$ & $\begin{array}{l}\text { Brand } 1 \\
\text { Brand } 2 \\
\text { Brand } 3\end{array}$ & $\begin{array}{l}0.65 \\
0.46 \\
4 \cdot 11\end{array}$ & $\begin{array}{l}\text { Brand } 1 \dagger \\
\text { Brand } 2 \\
\text { Brand } 3\end{array}$ & $\begin{array}{l}5.23 \\
0.95 \\
0.95\end{array}$ \\
\hline $\begin{array}{l}\text { Pre-packaged } \\
\text { chocolate-filled or } \\
\text { covered cookies }\end{array}$ & $\begin{array}{l}\text { Brand } 1 \\
\text { Brand } 2 \\
\text { Brand 3† }\end{array}$ & $\begin{array}{r}4 \cdot 76 \\
0 \cdot 12 \\
12 \cdot 63\end{array}$ & Not available & & $\begin{array}{l}\text { Brand } 1 \\
\text { Brand 2† }\end{array}$ & $\begin{array}{l}0.66 \\
8.04\end{array}$ & $\begin{array}{l}\text { Brand } 1 \\
\text { Brand } 2\end{array}$ & $\begin{array}{l}1.05 \\
2.05\end{array}$ \\
\hline Snacks & $\begin{array}{l}\text { Brand } 1 \\
\text { Brand } 2 \\
\text { Brand } 3\end{array}$ & $\begin{array}{l}0.11 \\
0 \cdot 13 \\
0 \cdot 10\end{array}$ & $\begin{array}{l}\text { Brand } 1 \\
\text { Brand } 2 \\
\text { Brand } 3 \\
\text { Brand } 4\end{array}$ & $\begin{array}{l}0.12 \\
0.47 \\
0.33 \\
0.43\end{array}$ & $\begin{array}{l}\text { Brand } 1 \\
\text { Brand } 2 \\
\text { Brand } 3 \\
\text { Brand } 4\end{array}$ & $\begin{array}{l}0.66 \\
0.47 \\
0.49 \\
2.43\end{array}$ & $\begin{array}{l}\text { Brand } 1 \\
\text { Brand } 2 \\
\text { Brand } 3 \\
\text { Brand } 4\end{array}$ & $\begin{array}{l}0.37 \\
0.55 \\
0.36 \\
0.63\end{array}$ \\
\hline $\begin{array}{l}\text { Pre-packaged } \\
\text { pastries }\end{array}$ & $\begin{array}{l}\text { Butter crescent†, } \\
\text { Lard crescent§ } \\
\text { Puff/filo pastry snack\| }\end{array}$ & $\begin{array}{r}21 \cdot 20 \\
3.90 \\
0.61\end{array}$ & $\begin{array}{l}\text { Cream-filled } \\
\text { poundcake }\end{array}$ & 0.00 & $\begin{array}{l}\text { Doughnuts } \\
\text { Puff pastry†† }\end{array}$ & $\begin{array}{l}0 \cdot 72 \\
3 \cdot 21\end{array}$ & $\begin{array}{l}\text { Cinnamon rolls, } \\
\text { brand } 1 \dagger \\
\text { Muffins }\|\|\end{array}$ & $\begin{array}{l}5 \cdot 70 \\
0.54\end{array}$ \\
\hline Local fast foods & $\begin{array}{l}\text { Savoury turnovers } \\
\text { Fried milanesa chicken } \\
\text { French fries }\end{array}$ & $\begin{array}{l}0.21 \\
0.23 \\
0.35\end{array}$ & $\begin{array}{l}\text { Shoestring potatoes } 1 \\
\text { Shoestring potatoes } \\
\text { Farofa, brand } 1^{\star *}\end{array}$ & $\begin{array}{l}0.75 \\
0.54 \\
0.44\end{array}$ & $\begin{array}{l}\text { Potato turnoverł‡ } \\
\text { Fried tacos§§ } \\
\text { Hamburger }\end{array}$ & $\begin{array}{l}3 \cdot 52 \\
1 \cdot 13 \\
3 \cdot 96\end{array}$ & $\begin{array}{l}\text { Tacos pastorףף } \\
\text { Cheese quesadilla } \\
\text { Gordita }^{\star \star \star}\end{array}$ & $\begin{array}{l}0.83 \\
1.35 \\
0 \cdot 10\end{array}$ \\
\hline $\begin{array}{l}\text { French fries from } \\
\text { international } \\
\text { chains }\end{array}$ & $\begin{array}{l}\text { McDonald's } \\
\text { Burger King } \\
\text { Walmart }\end{array}$ & $\begin{array}{l}0.78 \\
0.64 \\
1.04\end{array}$ & McDonald's & $4 \cdot 38$ & $\begin{array}{l}\text { Burger King } \\
\text { McDonald's } \\
\text { Taco Bell } \\
\text { KFC }\end{array}$ & $\begin{array}{l}0.44 \\
2.01 \\
1.40 \\
1.84\end{array}$ & $\begin{array}{l}\text { Burger King } \\
\text { McDonald's } \\
\text { KFC }\end{array}$ & $\begin{array}{l}1 \cdot 81 \\
2 \cdot 40 \\
4 \cdot 50\end{array}$ \\
\hline
\end{tabular}

PAHO, Pan American Health Organization.

*Exceeds PAHO/WHO recommendation of $<2 \%$ total IP-TFA content in vegetable oils and spreadable fats.

†Exceeds PAHO/WHO recommendation of $<5 \%$ total IP-TFA content in processed foods.

¥Similar to butter croissant.

$\S$ Similar to croissant, made with lard.

$\|$ Librillos/puff and filo pastry.

IDeep-fried and prepared with wheat dough; filling commonly meat, cheese and/or vegetables.

${ }^{\star \star}$ Toasted cassava flour mixture.

$\dagger †$ Prusianos/puff pastry filled with whipped cream.

$\neq$ Deep-fried, corn dough with potato filling.

§Fried corn tortillas tightly rolled, stuffed with shredded beef.

|| || Muffins or mantecadas.

१ी१Corn tortillas filled with meat.

${ }^{\star * \star}$ Thick corn tortilla stuffed with various ingredients like cheese.

\section{Change of industrially produced trans-fatty acid content: period 2015-2016}

There was a notable and significant decrease in the content of IP-TFA in the foods that contained a high level of IP-TFA in 2011-2012 (Tables 3-6). The largest decrease $(P<0.01)$ was in Buenos Aires, where content of TFA went from $12 \cdot 6-34 \cdot 8 \%$ in $2011-2012$ to nearly $0 \%$ in 2015-2016 in the pre-selected brands and types of foods (Table 3).

In the period 2015-2016 in San José and Mexico City, the food industry significantly increased the content of palmitic acid (16:0) and oleic acid (cis 9-18:1) in foods and reduced IP-TFA content compared with the same foods in 2011-2012 (Tables 4 and 6). The increase of palmitic acid was significantly higher $(P<0 \cdot 01)$ in some brands of cookies with artificially flavoured cream filling and cookies covered or filled with chocolate.
In Rio de Janeiro, the content of palmitic (16:0), oleic (cis 9-18:1) and linoleic acids (cis 9, cis 12-18:2) in foods also increased significantly $(P<0.01)$ in contrast to the period 2011-2012 (Table 5).

\section{Discussion}

IP-TFA levels have been reduced significantly in commonly consumed foods in some Latin American cities. Some key reasons for the observed reformulations of foods to reduce IP-TFA content may include: national and transnational recommendations (such as PAHO/WHO), policies and legislation introduced during this time, voluntary reduction from the industry, intersectoral coordination and government-food industry collaborations, as well as global pressure, dialogue and marketing that was going on during these periods in the region ${ }^{(11)}$. 
Table 3 Change in the fatty acid profile observed in the period 2015-2016 in those foods that had a high content of industrially produced trans-fatty acids in the period 2011-2012: Argentina

\begin{tabular}{|c|c|c|c|c|c|c|}
\hline \multirow[b]{3}{*}{ Food } & \multirow[b]{3}{*}{ Fatty acids } & \multicolumn{4}{|c|}{$\begin{array}{l}\text { Observed values according to period of data } \\
\text { collection ( } \mathrm{g} / 100 \mathrm{~g} \text { food) }\end{array}$} & \multirow[b]{3}{*}{$P$ value* } \\
\hline & & \multicolumn{2}{|c|}{ 2011-2012 } & \multicolumn{2}{|c|}{ 2015-2016 } & \\
\hline & & Mean & SD & Mean & SD & \\
\hline \multirow{10}{*}{$\begin{array}{l}\text { Pre-packaged chocolate-filled or } \\
\text { covered cookies }\end{array}$} & Saturated & & & & & \\
\hline & $12: 0$ & 0.213 & 0.001 & 1.684 & 0.002 & 0.0042 \\
\hline & $14: 0$ & 0.426 & 0.023 & 0.982 & 0.036 & 0.0297 \\
\hline & $16: 0$ & $4 \cdot 726$ & 0.496 & 4.733 & 0.524 & 0.5424 \\
\hline & $18: 0$ & $3 \cdot 214$ & 0.022 & $6 \cdot 324$ & 0.012 & 0.0015 \\
\hline & Monounsaturated & & & & & \\
\hline & $\begin{array}{l}\text { Cis 9-18: } 1 \\
\text { Polyunsaturated }\end{array}$ & 5.572 & 0.053 & $8 \cdot 561$ & 0.048 & 0.0013 \\
\hline & $\begin{array}{l}\text { Cis } 9 \text {, cis 12-18: } \\
\text { Trans }\end{array}$ & 1.043 & 0.051 & 0.482 & 0.072 & 0.0271 \\
\hline & Total† & $2 \cdot 416$ & 0.081 & 1.023 & 0.023 & 0.0004 \\
\hline & $\%$ of total fatty acids & \multicolumn{2}{|c|}{$12 \cdot 63$} & \multicolumn{2}{|c|}{0.007} & 0.0000 \\
\hline \multirow[t]{10}{*}{ Regular margarine } & Saturated & & & & & \\
\hline & $12: 0$ & 0.000 & 0.000 & 3.581 & 0.015 & 0.0001 \\
\hline & $14: 0$ & 0.112 & 0.001 & 1.392 & 0.023 & 0.0327 \\
\hline & $16: 0$ & 8.934 & 0.094 & $9 \cdot 114$ & 0.072 & 0.0259 \\
\hline & $18: 0$ & 8.764 & 0.214 & 18.591 & 0.119 & 0.0008 \\
\hline & Monounsaturated & & & & & \\
\hline & $\begin{array}{l}\text { Cis 9-18: } 1 \\
\text { Polyunsaturated }\end{array}$ & $19 \cdot 658$ & 0.016 & 9.914 & 0.256 & 0.0003 \\
\hline & $\begin{array}{l}\text { Cis } 9 \text {, cis 12-18: } 2 \\
\text { Trans }\end{array}$ & 13.963 & 0.097 & $25 \cdot 783$ & 0.185 & 0.0005 \\
\hline & Total† & \multicolumn{2}{|c|}{$29 \cdot 427$} & \multicolumn{2}{|c|}{0.000} & 0.0002 \\
\hline & $\%$ of total fatty acids & \multirow{2}{*}{\multicolumn{2}{|c|}{$34 \cdot 80$}} & \multirow{2}{*}{\multicolumn{2}{|c|}{0.000}} & 0.0000 \\
\hline \multirow[t]{9}{*}{ Butter crescent } & Saturated & & & & & \\
\hline & $12: 0$ & 0.000 & 0.000 & 0.391 & 0.017 & 0.0032 \\
\hline & $14: 0$ & 0.421 & 0.062 & 0.182 & 0.058 & 0.0783 \\
\hline & $16: 0$ & 5.283 & 0.047 & $2 \cdot 132$ & 0.009 & 0.0216 \\
\hline & $18: 0$ & 3.628 & 0.059 & $6 \cdot 744$ & 0.067 & 0.0328 \\
\hline & Monounsaturated & & & & & \\
\hline & $\begin{array}{l}\text { Cis 9-18: } 1 \\
\text { Polyunsaturated }\end{array}$ & $5 \cdot 173$ & 0.194 & $9 \cdot 877$ & 0.268 & 0.0012 \\
\hline & $\begin{array}{l}\text { Cis } 9 \text {, cis 12-18:2 } \\
\text { Trans }\end{array}$ & $2 \cdot 354$ & 0.194 & 1.647 & 0.246 & 0.0226 \\
\hline & Total† & \multicolumn{2}{|c|}{21.20} & 1.907 & 0.246 & 0.0002 \\
\hline
\end{tabular}

${ }^{*} P$ value for the difference between the periods 2011-2012 and 2015-2016 using the sample-paired $t$ test. †Defined as the sum of all positional isomers of trans-18:1 and trans-18:2.

Since the 2000s, in light of the overwhelming scientific evidence and growing public awareness about the adverse effects of consuming TFA, many multinational companies have switched sources of fat to reduce TFA levels in products. These changes have, however, been most pronounced in high-income countries ${ }^{(21)}$. For example, major food producers and manufacturers in the EU have voluntarily reformulated their products and the level of TFA has decreased significantly over the past 10 years in many EU Member States without the need for regulation ${ }^{(22)}$. The changes made to products sold in low- and middle-income countries have been less consistent $^{(21)}$. Our results show that, at least in the studied cities (a mix of middle- and high-income countries), transnational fast-food chains had already reformulated their products before legislation was passed. This is particularly true for the oil used for frying French fries during the period 2011-2012. For example, in Costa Rica, where mandatory legislation does not exist, the industry voluntarily reformulated cooking oils by replacing IP-TFA with a mixture of cis-unsaturated and saturated fatty acids. A similar phenomenon happened in Argentina during the 2004-2006 period before any legislation or recommendation was passed in the country ${ }^{(23)}$. Some authors have suggested that international dialogues and reconfigurations could have pressured more local industries to reformulate products, or otherwise their products would be non-competitive in international markets ${ }^{(24)}$.

Nutrition labelling is another approach that has been used to increase consumer knowledge and public awareness about the adverse health effects of TFA. The approach seems to have worked when governments mandate nutrition labelling and the industry voluntarily reduces the TFA content of its foodstuffs. For example, results from Canada suggest that mandatory labelling and the publicity regarding the harmful effects of TFA had an immediate effect on reducing the amount of TFA in the food supply ${ }^{(5)}$. In Australia and New Zealand, which 
Table 4 Change in the fatty acid profile observed in the period 2015-2016 in those foods that had a high content of industrially produced trans-fatty acids in the period 2011-2012: Costa Rica

\begin{tabular}{|c|c|c|c|c|c|c|}
\hline \multirow[b]{3}{*}{ Food } & \multirow[b]{3}{*}{ Fatty acids } & \multicolumn{4}{|c|}{$\begin{array}{l}\text { Observed values according to period of data } \\
\text { collection ( } \mathrm{g} / 100 \mathrm{~g} \text { food) }\end{array}$} & \multirow[b]{3}{*}{$P$ value } \\
\hline & & \multicolumn{2}{|c|}{ 2011-2012 } & \multicolumn{2}{|c|}{ 2015-2016 } & \\
\hline & & Mean & SD & Mean & SD & \\
\hline \multirow{10}{*}{$\begin{array}{l}\text { Pre-packaged chocolate-filled or } \\
\text { covered cookies, brand } 2\end{array}$} & Saturated & & & & & \\
\hline & $12: 0$ & 0.048 & 0.104 & 2.743 & 0.022 & 0.0006 \\
\hline & $14: 0$ & 1.571 & 0.113 & 1.343 & 0.013 & 0.1273 \\
\hline & $16: 0$ & 7.783 & 0.181 & 10.992 & 0.042 & 0.0006 \\
\hline & $18: 0$ & 2.919 & 0.105 & 1.438 & 0.011 & 0.0239 \\
\hline & Monounsaturated & & & & & \\
\hline & $\begin{array}{c}\text { Cis 9-18: } 1 \\
\text { Polyunsaturated }\end{array}$ & $6 \cdot 715$ & 0.226 & $7 \cdot 840$ & 0.217 & 0.0023 \\
\hline & $\begin{array}{l}\text { Cis } 9 \text {, cis 12-18: } 2 \\
\text { Trans }\end{array}$ & $1 \cdot 312$ & 0.218 & 1.626 & 0.013 & 0.0738 \\
\hline & Total† & 1.992 & 0.042 & 0.169 & 0.001 & 0.0012 \\
\hline & $\%$ of total fatty acids & \multicolumn{2}{|c|}{8.04} & \multicolumn{2}{|c|}{0.650} & 0.0010 \\
\hline \multirow[t]{10}{*}{ Light margarine } & Saturated & & & & & \\
\hline & $12: 0$ & 0.057 & 0.003 & 0.078 & 0.001 & 0.0894 \\
\hline & $14: 0$ & 0.166 & 0.011 & 0.174 & 0.001 & 0.0749 \\
\hline & $16: 0$ & $6 \cdot 249$ & 0.146 & 9.009 & 0.018 & 0.0021 \\
\hline & $18: 0$ & $2 \cdot 177$ & 0.068 & $2 \cdot 130$ & 0.006 & 0.1853 \\
\hline & Monounsaturated & & & & & \\
\hline & $\begin{array}{l}\text { Cis } 9-18: 1 \\
\text { Polyunsaturated }\end{array}$ & $10 \cdot 557$ & 0.259 & $13 \cdot 038$ & 0.032 & 0.0006 \\
\hline & $\begin{array}{l}\text { Cis } 9 \text {, cis 12-18:2 } \\
\text { Trans }\end{array}$ & 9.918 & 0.381 & $10 \cdot 873$ & 0.081 & $0 \cdot 7627$ \\
\hline & Total† & 0.884 & 0.002 & 0.567 & 0.002 & 0.0031 \\
\hline & $\%$ of total fatty acids & \multicolumn{2}{|c|}{$2 \cdot 25$} & \multicolumn{2}{|c|}{1.420} & 0.0001 \\
\hline
\end{tabular}

${ }^{*} P$ value for the difference between the periods 2011-2012 and 2015-2016 using the sample-paired $t$ test.

†Defined as the sum of all positional isomers of trans-18:1 and trans-18:2.

require TFA declaration on the nutrition label only when certain nutrition content and health claims are made, the industry has a variety of programmes and initiatives in place to reduce TFA in the food supply, and TFA content in table spreads, edible fats and oils for baking and various food items have been reduced without the need for regulatory measures in addition to existing labelling requirements ${ }^{(25)}$. In our results, Costa Rica and Mexico (both have mandatory nutrition labelling only if the food makes a claim about its fat or cholesterol content) saw reductions in the IP-TFA content of margarines to less than $2 \%$ of total fat, as well as in all varieties of sweet and salty snacks and fast foods to less than $5 \%$, or in some cases to less than $2 \%$ of total fat.

However, the voluntary reformulations have not been systematic or consistent across all products and in all countries $^{(3)}$ and this is perhaps where normative guidelines to regularize the content of IP-TFA could have led to the results observed. The case of Buenos Aires suggests this: there was high variability in IP-TFA content in foods sampled in 2011-2012, prior to any strict government regulation. In 2014, a regulation of IP-TFA content became effective in Argentina ${ }^{(23)}$. The data presented in the current study indicate that after this regulation and during the period 2015-2016 the content of IP-TFA across foods did not exceed the lower limit of TFA mandated by the regulation. There are several examples across the globe (mostly high-income countries) concerning regulations that have virtually eliminated IP-TFA by establishing an upper limit in its content. Denmark, for example, was able to virtually eliminate IP-TFA from the food supply by banning these fatty acids ${ }^{(5)}$. New York City has been restricting IP-TFA since 2007 in restaurants, leading to a virtual elimination of IP-TFA content among foods served by major restaurant chains in the $\mathrm{USA}^{(8)}$. Before the ban, the city had worked with the restaurant industry, which supported a voluntary initiative, to minimize the IP-TFA content. Similar to the case in Latin America with major food companies ${ }^{(3)}$, these voluntary efforts by the food industry did not result in measurable changes and were deemed insufficient to eliminate the health risk $^{(8)}$. Our findings thus add to the existing literature ${ }^{(7-9,26)}$ that regulations that establish an upper limit of IP-TFA can produce more favourable and consistent results of product reformulation across foodstuffs.

It is also important to consider how products have been reformulated because that affects the nutrition (or in this case, fatty acid) profile of the new foodstuffs and, ultimately, the health of their consumers. Our data show that for the period 2015-2016, the removal of TFA came at the expense of an increase in SFA, particularly in San José, Mexico City and Rio de Janeiro. This contrasts with previous reports that saturated fat content was mostly unchanged after product reformulation - although 
Table 5 Change in the fatty acid profile observed in the period 2015-2016 in those foods that had a high content of industrially produced trans-fatty acids in the period 2011-2012: Brazil

\begin{tabular}{|c|c|c|c|c|c|c|}
\hline \multirow[b]{3}{*}{ Food } & \multirow[b]{3}{*}{ Fatty acids } & \multicolumn{4}{|c|}{$\begin{array}{l}\text { Observed values according to period of data } \\
\text { collection ( } \mathrm{g} / 100 \mathrm{~g} \text { food) }\end{array}$} & \multirow[b]{3}{*}{$P$ value ${ }^{\star}$} \\
\hline & & \multicolumn{2}{|c|}{ 2011-2012 } & \multicolumn{2}{|c|}{ 2015-2016 } & \\
\hline & & Mean & SD & Mean & SD & \\
\hline \multirow{10}{*}{$\begin{array}{l}\text { Pre-packaged artificially flavoured } \\
\text { cream-filled cookies, brand } 3\end{array}$} & Saturated & & & & & \\
\hline & $12: 0$ & 0.053 & 0.023 & 0.642 & 0.012 & 0.0468 \\
\hline & $14: 0$ & 0.042 & 0.012 & 0.471 & 0.001 & 0.0473 \\
\hline & $16: 0$ & $2 \cdot 746$ & 0.017 & 11.582 & 0.103 & 0.0001 \\
\hline & $18: 0$ & 1.957 & 0.032 & 2.359 & 0.028 & 0.0283 \\
\hline & Monounsaturated & & & & & \\
\hline & $\begin{array}{c}\text { Cis 9-18: } 1 \\
\text { Polyunsaturated }\end{array}$ & $4 \cdot 331$ & 0.026 & $9 \cdot 679$ & 0.126 & 0.0001 \\
\hline & $\begin{array}{l}\text { Cis } 9 \text {, cis } 12-18: 2 \\
\text { Trans }\end{array}$ & 0.074 & 0.001 & 4.001 & 0.036 & 0.0001 \\
\hline & Total† & 3.872 & 0.361 & 0.400 & 0.003 & 0.0001 \\
\hline & $\%$ of total fatty acids & \multicolumn{2}{|c|}{$23 \cdot 30$} & \multicolumn{2}{|c|}{0.120} & 0.0000 \\
\hline \multirow[t]{10}{*}{ Regular margarine, brand 3} & Saturated & & & & & \\
\hline & $12: 0$ & 0.923 & 0.092 & 7.474 & 0.017 & 0.0001 \\
\hline & $14: 0$ & 0.453 & 0.031 & $2 \cdot 810$ & 0.018 & 0.0158 \\
\hline & $16: 0$ & $10 \cdot 171$ & 0.598 & $18 \cdot 333$ & 0.126 & 0.0001 \\
\hline & $18: 0$ & $5 \cdot 876$ & 0.073 & 11.968 & 0.132 & 0.0001 \\
\hline & Monounsaturated & & & & & \\
\hline & $\begin{array}{l}\text { Cis 9-18: } 1 \\
\text { Polyunsaturated }\end{array}$ & 14.751 & 0.568 & $23 \cdot 279$ & 0.261 & 0.0001 \\
\hline & $\begin{array}{l}\text { Cis } 9 \text {, cis 12-18:2 } \\
\text { Trans }\end{array}$ & $16 \cdot 951$ & 0.631 & 20.544 & 0.873 & 0.0001 \\
\hline & Total† & $5 \cdot 727$ & 0.831 & $4 \cdot 143$ & 0.032 & 0.0001 \\
\hline & $\%$ of total fatty acids & \multicolumn{2}{|c|}{9.46} & \multicolumn{2}{|c|}{$4 \cdot 140$} & 0.0011 \\
\hline
\end{tabular}

${ }^{*} P$ value for the difference between the periods 2011-2012 and 2015-2016 using the sample-paired $t$ test.

†Defined as the sum of all positional isomers of trans-18:1 and trans-18:2.

the evidence from that report came from high-income, Western countries ${ }^{(27)}$. By contrast, in Argentina, IP-TFA were replaced with oleic and linoleic acids. According to Menaa et $a l .{ }^{(28)}$, the three industrial strategies used to reduce TFA in processed foods are: (i) food reformulation; (ii) modification of fatty acid composition by fat interesterification; and (iii) genetic modification of fatty acids' composition. These strategies may vary widely depending on the availability and costs of various other fats and adoption of food technologies ${ }^{(5)}$. For example, Lima et al. (unpublished results) suggested that in Brazil the supply of new technological alternatives, such as interesterification, contributed to the reduction of IP-TFA content observed in margarine and chocolate-filled cookies during the period 2015-2016. In Argentina, the notable reduction of IP-TFA evidenced in the period 2015-2016 in Buenos Aires coincides with an increase in the use of hybrid oils of plant species (i.e. high-oleic sunflower oil) to produce oils with high stearic acid (18:0), oleic acid (cis 9-18:1) and linoleic acid (cis 9, cis 12-18:2), as the content of these fatty acids was significantly greater $(P<0.05)$ than evidenced for the same foods in the period 2011-2012. Therefore, working with the industry on the adoption of reformulation technology and techniques seems critical to achieve a more favourable nutrition profile for the foods.
In the case of IP-TFA it is evident that replacement of these TFA with either polyunsaturated or saturated fatty acids will lead to a net health benefit compared with no replacement at all. Using data from two of the largest prospective cohort studies, Mozaffarian and Clarke showed that for each $2 \%$ energy replacement of TFA with saturated fats, the CHD risk was $17 \%$ lower ${ }^{(29)}$. Likewise, in controlled trials, each $1 \%$ energy replacement of TFA with saturated fats has been associated with a significant decrease in CHD biomarkers $^{(29)}$. More recent epidemiological evidence suggests a relatively neutral effect of total saturated fat on $\mathrm{CHD}^{(30)}$ and a slightly beneficial effect when it is compared with the consumption of refined carbohydrates and sugars $^{(30-32)}$. That is, there is a net health benefit even when replacing IP-TFA with tropical oils (rich in SFA) ${ }^{(29)}$, although benefits are projected to be greatest when vegetable oils with high unsaturated fatty acid content replace the IP-TFA.

Our study design has both limitations and strengths. The current research is the first to show the elimination of TFA in the food supply in four Latin American cities. Although the results cannot be generalized to the entire countries, our study provides an overview of the IP-TFA content in foods commonly consumed in some of the most populated cities in these countries. The study is descriptive in nature and therefore is not a rigorous evaluation of policy or programmes because it lacks control groups and process 
Table 6 Change in the fatty acid profile observed in the period 2015-2016 in those foods that had a high content of industrially produced trans-fatty acids in the period 2011-2012: Mexico

\begin{tabular}{|c|c|c|c|c|c|c|}
\hline \multirow[b]{3}{*}{ Food } & \multirow[b]{3}{*}{ Fatty acids } & \multicolumn{4}{|c|}{$\begin{array}{l}\text { Observed values according to period of data } \\
\text { collection ( } \mathrm{g} / 100 \mathrm{~g} \text { food) }\end{array}$} & \multirow[b]{3}{*}{$P$ value } \\
\hline & & \multicolumn{2}{|c|}{ 2011-2012 } & \multicolumn{2}{|c|}{ 2015-2016 } & \\
\hline & & Mean & SD & Mean & SD & \\
\hline \multirow{10}{*}{$\begin{array}{l}\text { Pre-packaged artificially flavoured cream-filled } \\
\text { cookies, brand } 1\end{array}$} & Saturated & & & & & \\
\hline & $12: 0$ & 0.291 & 0.016 & 1.269 & 0.021 & 0.0115 \\
\hline & $14: 0$ & 0.579 & 0.043 & 0.657 & 0.003 & 0.0724 \\
\hline & $16: 0$ & 10.459 & 0.116 & $18 \cdot 185$ & 0.256 & 0.0001 \\
\hline & $18: 0$ & 3.303 & $0 \cdot 152$ & $2 \cdot 242$ & 0.013 & 0.0753 \\
\hline & Monounsaturated & & & & & \\
\hline & $\begin{array}{l}\text { Cis 9-18: } 1 \\
\text { Polyunsaturated }\end{array}$ & $9 \cdot 332$ & 0.037 & 14.083 & 0.278 & 0.0012 \\
\hline & $\begin{array}{l}\text { Cis } 9 \text {, cis } 12-18: 2 \\
\text { Trans }\end{array}$ & 2.535 & 0.064 & 0.063 & 0.008 & 0.0167 \\
\hline & Total† & $2 \cdot 315$ & 0.031 & 1.018 & 0.011 & 0.0011 \\
\hline & $\%$ of total fatty acids & \multicolumn{2}{|c|}{5.23} & \multicolumn{2}{|c|}{3.690} & 0.0001 \\
\hline \multirow[t]{10}{*}{ Cinnamon rolls, brand 1} & Saturated & & & & & \\
\hline & $12: 0$ & 0.018 & 0.008 & 0.077 & 0.032 & 0.7321 \\
\hline & $14: 0$ & 0.069 & 0.022 & $0 \cdot 101$ & 0.004 & 0.0875 \\
\hline & $16: 0$ & 1.894 & 0.014 & 3.333 & 0.282 & 0.0016 \\
\hline & $18: 0$ & 0.562 & 0.014 & 0.768 & 0.007 & 0.0875 \\
\hline & Monounsaturated & & & & & \\
\hline & $\begin{array}{l}\text { Cis } 9-18: 1 \\
\text { Polyunsaturated }\end{array}$ & $1 \cdot 257$ & 0.086 & $2 \cdot 726$ & 0.011 & 0.0217 \\
\hline & $\begin{array}{l}\text { Cis } 9 \text {, cis 12-18: } 2 \\
\text { Trans }\end{array}$ & 2.990 & 0.059 & 1.959 & 0.004 & 0.0328 \\
\hline & Total† & 0.521 & 0.028 & 0.08 & 0.002 & 0.0118 \\
\hline & $\%$ of total fatty acids & \multicolumn{2}{|c|}{5.70} & \multicolumn{2}{|c|}{0.940} & 0.0000 \\
\hline
\end{tabular}

${ }^{*} P$ value for the difference between the periods 2011-2012 and 2015-2016 using the sample-paired $t$ test.

†Defined as the sum of all positional isomers of trans-18:1 and trans-18:2.

measures, and includes data only during the time that the reduction of IP-TFA was already taking place, but it does allow to generate a discussion about the potential impact created by the different strategies that were introduced by countries during the period observed. Another weakness is that the primary data were gathered during the time when the reduction of TFA was taking place, so there is no information on fatty acid content prior to the reformulation. Finally, it is important to note that the reduction observed in the levels of TFA in foods does not guarantee intake below/ above dietary recommendations, especially in vulnerable pockets of the population that were not assessed in our study. Therefore, the findings of the present research should be complemented with the promotion of several key dietary priorities for cardiometabolic health. In sum, the reduction of IP-TFA in the foods analysed suggests that the strategies implemented by the public health authorities, the voluntary or mandatory food reformulation made by the food industry and cooking oil companies to their products, could potentially have influenced the elimination of IP-TFA. The experiences gained and lessons learned throughout this process could be applied to other health initiatives included in the WHO Global Action Plan for the Prevention and Control of Noncommunicable Diseases 2013-2020 (33). For example, cross-sector initiatives to reduce sugars, sodium and saturated fat in foods can learn from the experience of
IP-TFA in terms of government involvement in the nutrition labelling and education/awareness efforts. These educational initiatives could pressure the food industry into reformulating its products if consumers are aware and demand these reformulations. Similar to the process of identifying alternatives to TFA, the food industry will often lead the study of the organoleptic characteristics of the replacement and it will rest upon others, often intersectoral discussions involving academia, government, consumers and industry, to support the study of the health consequences (both positive and negative) of these replacements. For example, in the case of sugars, where the food industry is quickly moving towards replacement with artificial sweeteners ${ }^{(34)}$, there must be a surge in support of investigating the health consequences of these controversial additives ${ }^{(35)}$. Therefore, once product reformulations have been identified and their health benefits have been established, we advocate for continuous monitoring and surveillance for compliance, as well as evaluation of the cost-effectiveness of these strategies in light of the health and well-being outcomes that they are ultimately trying to achieve. Finally, our study underscores the need to monitor progress of these public health initiatives across the globe in a variety of income denominations, including highincome countries in various parts of the world, as well as low- and middle-income countries and in pockets of lowincome communities. It is especially important to continue to 
monitor exposure to unformulated products among underserved communities, as well as long-term exposure to the newly reformulated products.

\section{Acknowledgements}

Acknowledgements: The authors would like to thank Dr Daniel Ferrante for his help in coordinating the study in Buenos Aires, Argentina. Financial support: The present work was partially supported by PAHO/WHO (grant number HSD-S03-309-PG/10-11/999 (GD 1603)). Additional funding came from the Fundação Carlos Chagas Filho de Amparo à Pesquisa do Estado do Rio de Janeiro (FAPERJ), Brazil (grant number E-26/101.561/2010); the National Ministry of Health, Argentina; Cátedra Bromatología y Nutrición, Facultad de Bioquímica y Ciencias Biológicas, Universidad Nacional del Litoral, Santa Fe, Argentina; Centro de Investigación en Nutrición y Salud, Instituto Nacional de Salud Pública, México; and from the Costa Rican Institute for Research and Education on Nutrition and Health (INCIENSA). The funders had no role in the design, analysis or writing of this article. Conflict of interest: The authors do not have any actual or potential conflict of interest, including any financial, personal or other relationship with other people or organizations, within 6 years of beginning this work that could inappropriately influence, or be perceived to influence, the work. Authorship: R.M.-R. conceived the study, study design and analysis, led the writing and analysis of the data, and led the drafting of revisions contributing to interpretation. U.C.-R. assisted with data analysis and contributed significantly to the writing and interpretation of results. E.J. contributed to the writing and interpretation of results. T.A., M.d.G.T.d.C., S.V. and C.B. contributed significantly in the homologation of analytical techniques among the participant laboratories, led the determination of TFA in foods and contributed to the interpretation of results and writing. All authors contributed significantly to drafting the manuscript and revisions, and reviewed all drafts and the final manuscript. Ethics of human subject participation: In each city included in the multicentre study, the research protocol was approved by an Institutional Review Board before initiating data collection.

\section{References}

1. Food and Agriculture Organization of the United Nations (2010) Fats and Fatty Acids in Human Nutrition. Report of an Expert Consultation, 10-14 November 2008, Geneva. FAO Food and Nutrition Paper no. 91. Rome: FAO.

2. Academy of Nutrition and Dietetics (2014) Position of the Academy of Nutrition and Dietetics: dietary fatty acids for health adults. $J$ Acad Nutr Diet 114, 136-153.

3. Monge-Rojas R, Colón-Ramos U, Jacoby E et al. (2011) Voluntary reduction of trans-fatty acids in Latin American and the Caribbean: current situation. Rev Panam Salud Publica 29, 126-129.
4. World Health Organization (2003) Diet, Nutrition and the Prevention of Chronic Diseases. Report of a Joint WHO/FAO Expert Consultation. WHO Technical Report Series no. 916. Geneva: WHO.

5. L'Abbé MR, Stender S, Skeaff CM et al. (2009) Approaches to removing trans fats from the food supply in industrialized and developing countries. Eur J Clin Nutr 63, 50-67.

6. Tan AS (2009) A case study of the New York City trans-fat story for international application. J Public Health Policy 30, 3-16.

7. Leth T, Jensen HG, Mikkelsen AA et al. (2006) The effect of the regulation on trans fatty acid content in Danish food. Atheroscler Suppl 7, 53-56.

8. Angell SY, Cobb LK, Curtis CJ et al. (2012) Change in trans fatty acid content of fast-food purchases associated with New York City's restaurant regulation: a pre-post study. Ann Intern Med 157, 81-86.

9. Mozaffarian D, Jacobson MF \& Greenstein JS (2010) Food reformulations to reduce trans fatty acids. New Engl J Med 362, 2037-2039.

10. Baylin A, Siles X, Donovan-Palmer A et al. (2007) Fatty acid composition of Costa Rican foods including trans fatty acid content. J Food Compost Anal 20, 182-192.

11. Colón-Ramos U, Monge-Rojas R \& Campos H (2014) Impact of WHO recommendations to eliminate industrial trans-fatty acids from the food supply in Latin America and the Caribbean. Health Policy Plan 29, 529-541.

12. Pan American Health Organization (2007) PAHO/WHO Task Force Trans Fats Free Americas: Conclusions and Recommendations, April 26-27, 2007, Washington, DC. http://www.sld.cu/ galerias/pdf/sitios/diabetes/final_paho_who_task_force_ conclusions_may_17_07.pdf (accessed April 2017).

13. Pan American Health Organization (2008) Trans Fat Free Americas: Declaration of Rio de Janeiro. http://www.paho. org/hq/index.php?option=com_docman\&task=doc_view\& gid=13430\&Itemid=270 (accessed April 2017).

14. Secretaría de Salud de México (2010) Norma Oficial Mexicana NOM-051-SCFI/SSA1-2010. Especificaciones generales de etiquetado para alimentos y bebidas no alcohólicas preenvasados - Información comercial y sanitaria. Diario Oficial de la Federación. http://www.dof.gob.mx/nota_detalle_popup. php?codigo $=5137518$ (accessed April 2017).

15. Consejo de Ministros de Integración Económica Centroamericana (2012) Reglamento técnico centroamericano RTCA 67.01.60:10: etiquetado nutricional de productos alimenticios preenvasados para consumo humano para la población a partir de 3 años de edad. https://extranet.who.int/nutrition/gina/sites/ default/files/COMIECO\%202011\%20Etiquetado\%20Nutricional $\% 20$ de $\% 2$ Productos $\% 20$ Alimenticios $\% 20$ Preenvasados $\%$ 20para\%20Consumo\%20Humano.pdf (accessed April 2017).

16. Secretaría de Políticas Regulación de Institutos, Secretaría de Agricultura Ganadería y Pesca de Argentina (2010) Código Alimentario Argentino. Resolución Conjunta 137/2010 y 941/ 2010. http://servicios.infoleg.gob.ar/infolegInternet/anexos/ 175000-179999/176669/norma.htm (accessed April 2017).

17. Monge-Rojas R, Campos H \& Jacoby E (2010) Determination of the trans fatty acid content of common processed foods and the plasma fatty acid profile of Latin American and Caribbean urban populations: multicentric collaborative study sponsored by WHO/PAHO 2010. Protocol. http://www.inciensa.sa.cr/ investigacion/Protocol\%20Multicentric\%20Study.pdf (accessed April 2017).

18. International Organization for Standardization (1997) Guide 43-1. Proficiency testing by interlaboratory comparisons Part 1: Development and operation of proficiency testing schemes. ISO/IEC Guide 43-1:1997(E). http://www.iecnormen.de/dokumente/preview-pdf/info_isoiecguide43-1\% 7Bed2.0\%7Den.img.pdf (accessed April 2017). 
19. International Organization of Legal Metrology (2004) Quantity of product in prepackages International Recommendation. OIML R 87. https://www.oiml.org/en/files/pdf_r/r087-e04.pdf (accessed April 2017).

20. Association of Official Analytical Chemists (2010) Official Methods of Analysis of AOAC International: Current Through Revision 3, 18th ed. Gaithersburg, MD: AOAC.

21. Downs SM, Singh A, Gupta V et al. (2015) The need for multisectoral food chain approaches to reduce trans-fat consumption in India. BMC Public Health 15, 693.

22. World Health Organization (2015) Eliminating Trans Fats in Europe. A Policy Brief. http://www.euro.who.int/_data/ assets/pdf_file/0010/288442/Eliminating-trans-fats-in-Europe-Apolicy-brief.pdf?ua=1 (accessed April 2017).

23. Rubinstein A, Elorriaga N, Garay OU et al. (2015) Eliminating artificial trans fatty acids in Argentina: estimated effects on the burden of coronary heart disease and costs. Bull World Health Organ 93, 614-622.

24. Hendry VL, Almíron-Roig E, Monsivais P et al. (2015) Impact of regulatory interventions to reduce intake of artificial trans-fatty acids: a systematic review. Am J Public Health $\mathbf{1 0 5}$, $32-42$.

25. Reuss R, McKague K, Webb T et al. (2009) Cardiovascular health: trans fatty acids in Australia and New Zealand. Australas Epidemiol 16, 20-22.

26. Menaa F, Menaa A, Menaa B et al. (2013) Trans-fatty acids, dangerous bonds for health? A background review paper of their use, consumption, health implications and regulation in France. Eur J Nutr 52, 1289-1302.
27. Downs SM, Thow AM \& Leeder SR (2013) The effectiveness of policies for reducing dietary trans fat: a systematic review of the evidence. Bull World Health Organ 91, 262-269.

28. Menaa F, Menaa A, Tréton J et al. (2013) Technological approaches to minimize industrial trans fatty acids in foods. J Food Sci 78, 377-386.

29. Mozaffarian D \& Clarke R (2009) Quantitative effects on cardiovascular risk factors and coronary heart disease risk of replacing partially hydrogenated vegetable oils with other fats and oils. Eur J Clin Nutr 63, Suppl. 2, S22-S33.

30. Mozaffarian D (2016) Dietary and policy priorities for cardiovascular disease, diabetes, and obesity. Circulation 133, 187-225.

31. Jakobsen MU, O'Reilly EJ, Heitmann BL et al. (2009) Major types of dietary fat and risk of coronary heart disease: a pooled analysis of 11 cohort studies. Am J Clin Nutr 89, 1425-1432.

32. Jakobsen MU, Dethlefsen C, Joensen AM et al. (2010) Intake of carbohydrates compared with intake of saturated fatty acids and risk of myocardial infarction: importance of the glycemic index. Am J Clin Nutr 91, 1764-1768.

33. World Health Organization (2013) Global Action Plan for the Prevention and Control of Noncommunicable Diseases 2013-2020. WHO Geneva.

34. Shankar P, Ahuja S \& Sriram K (2013) Non-nutritive sweeteners: review and update. Nutrition 29, 1293-1299.

35. Borges MC, Louzada ML, de Sá TH et al. (2017) Artificially sweetened beverages and the response to the global obesity crisis. PLoS Med 14, e1002195. 